\title{
Generation of Low-Frequency Kinetic Waves at the Footpoints of Pre-Flare Coronal Loops
}

\author{
Alexandr Kryshtal ${ }^{1}$ Anna Voitsekhovska ${ }^{1}$. \\ Oleg Cheremnykh ${ }^{1}$ - Istvan Ballai ${ }^{2}$ Gary Verth ${ }^{2}$. \\ Viktor Fedun ${ }^{3}$ (D)
}

Received: 11 May 2020 / Accepted: 20 October 2020 / Published online: 24 November 2020

(C) The Author(s) 2020

\begin{abstract}
In this study we discuss the excitation of low-frequency plasma waves in the lower-middle chromosphere region of loop footpoints for the case when the plasma can be considered to be in a pre-flare state. It is shown that among the well-known semi-empirical models of the solar atmosphere, only the VAL (F) model together with a particular set of basic plasma parameters and amplitudes of the electric and magnetic fields supports generation of low-frequency wave instability. Our results show that it is possible to predict the onset of the flare process in the active region by using the interaction of kinetic Alfvén and kinetic ion-acoustic waves, which are solutions of the derived dispersion equation. The VAL (F) model allows situations when the main source of the aforementioned instability can be a sub-Dreicer electric field and drift plasma movements due to presence of spatial inhomogeneities. We also show that the generation of kinetic Alfvén and kinetic ion-acoustic waves can occur both, in plasma with a purely Coulomb conductivity and in the presence of small-scale Bernstein turbulence. The excitation of the small amplitude kinetic waves due to the development of low threshold instability in plasma with relatively low values of the magnetic field strength is also discussed.
\end{abstract}

Keywords Plasma instabilities · Solar atmosphere · Flares

V. Fedun

v.fedun@sheffield.ac.uk

A. Kryshtal

alexandr.kryshtal@gmail.com

I. Ballai

I.Ballai@sheffield.ac.uk

G. Verth

G.Verth@sheffield.ac.uk

1 Space Research Institute, Kiev, 03680, 187, Ukraine

2 Plasma Dynamics Group, School of Mathematics and Statistics, The University of Sheffield, Hicks Building, Hounsfield Road, Sheffield S3 7RH, UK

3 Plasma Dynamics Group, Department of Automatic Control and Systems Engineering, The University of Sheffield, Mappin Street, Sheffield, S1 3JD, UK 


\section{Introduction}

High-resolution solar observational data obtained during Hinode, SDO, STEREO, IRIS missions have confirmed that the coronal heating problem will most likely only be solved if the photosphere, chromosphere, transition region and corona are considered together as a connected energetic circuit (see, e.g., Aschwanden, 2001, 2005). In this regard present theoretical models that attempt to explain the problem of coronal heating have a number of limitations as regards their applicability. These limitations are related to the spatial inhomogeneity of the plasma (see, e.g., Aschwanden, 2001, 2005; Heinzel, Dorotovič, and Rutten, 2007; Reale, 2010), the location of the regions of the primary energy release of some flares in active regions (ARs) (Reznikova et al., 2009; Zaitsev, Stepanov, and Tsap, 1994; Charikov and Shabalin, 2015), anomalously low temperatures of individual flares (Shchukina and Trujillo Bueno, 2013; Benka, 1994; Somov, 1994) and a number of other observations. These issues are covered in detail in our previous work (Kryshtal, Voitsekhovska, and Gerasimenko, 2019).

Most solar flare models predict the appearance of DC electric fields that provide the simplest and most obvious mean to accelerate electrons. When a thermal plasma is under the influence of an electric field, a fraction of the electrons that have speeds greater than a critical speed, $v_{c}$, will be freely accelerated out of the thermal distribution (Dreicer, 1959, 1960). The critical speed is determined by the temperature of electrons, their number density, electric field strength and the resistivity of the plasma. Holman (1985) estimated that the maximum number of electrons that can be accelerated is given by

$$
N \leq 4.3 \times 10^{29}\left(\frac{B}{100 \mathrm{mT}}\right) \frac{A}{5.3 \times 10^{17} \mathrm{~cm}^{2}}\left(\frac{10^{7} \mathrm{~K}}{T}\right)^{1 / 2},
$$

where $A$ is the area of the current sheet that is associated with the process of magnetic reconnection during a flare process.

To find evidence of the presence of large-scale quasi-static weak electric fields in the solar atmosphere, Foukal and Hinata (1991) proposed using additional Stark broadening (Griem, 1974) of Balmer lines $H_{\beta}$ with $N \geq 8$. Indeed, their proposition has now been confirmed, and several indirect evidence of electric current heating the plasma via Joule dissipation have been adduced. This evidence includes the additional footpoint emission of flare loops observed during microwave spikes; extended thermal sources clearly seen in loops at coronal heights; a correlation between the shear of photospheric magnetic field with enhanced coronal heating and many others (see, e.g., Benka, 1994). Accordingly, a theoretical model has been developed (see, e.g., Kryshtal, Gerasimenko, and Voitsekhovska, 2012, 2014) to show how small-scale plasma waves can be generated in pre-flare AR loop structures due to the presence of a weak background electric field. Such fields are known as sub-Dreicer fields if their amplitudes are much less than the amplitude of the local Dreicer field (Miller et al., 1997). The importance of such fields resides in the fact that the existence of subDreicer fields in a loop can change the evolutionary process of a flare. Sub-Dreicer fields have successfully explained the hard X-ray spectra that is observed during flares, however, they cannot explain the existence of very energetic electrons due to the finite length of small electric field. More importantly, these weak electric fields are responsible for the production of runaway electrons. Direct sub-Deicer fields parallel to the magnetic field resulting from reconnecting field lines are possible candidates for explaining the impulsively accelerated electrons (see, e.g., Kuijpers, van der Post, and Slottje, 1981; Holman, 1985; MoghaddamTaaheri and Goertz, 1990; Miller et al., 1997; Xu and Emslie, 2007; Guo, Emslie, and Piana, 2013; Tsap and Kopylova, 2017; Zaitsev and Stepanov, 2018) 
According to Heyvaerts, Priest, and Rust (1977), the flaring process in an AR (loops, in particular) starts during a preheating phase, which precedes an impulsive phase and the plasma becomes visible in soft X-rays and extreme ultraviolet (EUV) wavelengths. The preheating phase begins as a result of the Buneman instability (see, e.g., Alexandrov, Bogdankevich, and Rukhadze, 1984; Chen, 1984), which appears when the electron drift velocity exceeds the electron thermal velocity. Therefore, sub-Dreicer electric fields play an important role in the excitation of various types of instabilities and hence in the generation of small-scale plasma turbulence well before the preheating phase. The amplitudes of generated plasma waves during the linear stage of the initial perturbation do not exceed the level of thermal noise, but undamped waves can result in appearance of different types of three-wave interactions (see, e.g., Kryshtal and Gerasimenko, 2004; Kryshtal, Gerasimenko, and Voitsekhovska, 2012). This aspect might influence the process of three-wave interaction and can be used for the short-term flare prediction (Aurass, 1994). Among the great number of waves that can appear in a magneto-active plasma (Alexandrov, Bogdankevich, and Rukhadze, 1984), two in particular are of great interest, i.e. the kinetic Alfvén wave (KAW) and the kinetic ion-acoustic wave (KIAW). Both types of waves have unique properties (see, e.g., Hasegawa and Chen, 1976a) and can effectively accelerate particles, (Miller et al., 1997), take part in processes of three-wave interaction (see, e.g., Hasegawa and Chen, 1976b; Brodin, Stenflo, and Shukla, 2006), support the development of turbulence (which is a necessary element in the process of formation of pre-flare current sheets) and can cause abnormal resistivity (Somov, 1994). In solar plasma studies all these processes and effects have been investigated in the post-flare stage of the plasma, without the presence of a background electric field, i.e. when beams of energetic particles are supposed to be the main driver of KAW's instability (see, e.g., Zharkova et al., 2011; Kumar, Nakariakov, and Cho, 2016; Druett et al., 2017). On the other hand, a large number of studies have been devoted to the study of KAW and plasma wave generation in solar atmosphere and solar flares; see e.g., Voitenko and Goossens (2002), Wu and Fang (2007), Tsiklauri (2011), Artemyev, Zimovets, and Rankin (2016).

The use of semi-empirical models of the flare chromosphere for AR forecasting may significantly increase its accuracy. However, this is possible only by taking into account a number of conditions, since the majority of these models describe the post-flare atmosphere. Therefore, only models which represent the quiet Sun atmosphere, e.g. VAL (F) type (see, e.g., Vernazza, Avrett, and Loeser, 1981) can be used to describe instabilities and corresponding wave generation in a pre-flare plasma. The use of MAVN (F1) (Machado et al., 1980) and FAL (PM) (Fontenla, Avrett, and Loeser, 1993) models, previously considered by Kryshtal, Voitsekhovska, and Gerasimenko (2019) for short-term flare prediction, are applicable only under rare conditions, for example, by taking into account the special role of energetic particle beams (see, e.g., Harra, Matthews, and Culhane, 2001). The generation of instabilities may also be suppressed by secondary beams and, therefore, the beam models may play only an additional role.

The present paper is devoted to the study of the pre-flare stage of the plasma by using the VAL (F) model, when the sub-Dreicer electric field acts as the main driver of the plasma instability that will drive KAWs and KIAWs without the need of energetic particle beams near loops' footpoints. Our approach follows closely the methodology developed recently by Kryshtal, Voitsekhovska, and Gerasimenko (2019). Our study can also be applied to the situation when flares occur in succession in the same AR, i.e. when the percentage of energetic particles after the first flare has become too small to influence the onset of the succeeding flare (see, e.g., Harra, Matthews, and Culhane, 2001). 


\section{Pre-Flare Plasma Model and Dispersion Relation}

It is well known that in a purely hydrogen plasma that is under the influence of an electric field, $E_{0}$, (with field strength less than the Dreicer field, $E_{D}$ ) parallel to the ambient magnetic field, a fraction of electrons with velocities greater than the critical value $\left(E_{c} / E_{0}\right)^{1 / 2} v_{T e}$ can overcome the drag force due to Coulomb collisions and become runaway electrons. Here $v_{T e}=\sqrt{k_{B} T_{e} / m_{e}}$ is the electron thermal speed, with $k_{B}$ the Boltzmann constant, $T_{e}$ the electron temperature, $m_{e}$ is the electron mass and the critical electric field is defined as

$$
E_{c} \approx 2 E_{D}=e k_{D}^{2} \Lambda_{c}=2 \times 10^{-13} \frac{N\left(\mathrm{~cm}^{-3}\right) \ln \Lambda_{c}}{T_{e}(\mathrm{eV})} .
$$

In the above expression $e$ is the electron charge, $k_{D}=\omega_{p e} / v_{T e}$ is the Debye wavelength, $\omega_{p e}$ is the electron plasma frequency, $N$ is the electron density, $T_{e}(\mathrm{eV})$ is the electron temperature measured in $\mathrm{eV}$, and $\ln \Lambda_{c}$ is the Coulomb logarithm estimated at the critical velocity. As the runaway electrons are further accelerated by the electric field, more electrons are introduced to the runaway region by collisions. As a result, the distribution function of electrons becomes distorted (with a runaway tail). We assume

$$
\left(\varepsilon_{\mathrm{R}}\right)_{\min } \ll \varepsilon_{\mathrm{R}} \equiv \frac{E_{0}}{E_{\mathrm{D}}} \ll\left(\varepsilon_{\mathrm{R}}\right)_{\max } \ll 1 .
$$

By taking into account the model assumptions presented by Kryshtal, Voitsekhovska, and Gerasimenko (2019) we consider the case when $T_{e} / T_{i}>1, v_{e i} \neq 0$ and $\varepsilon_{R} \neq 0$. Here, $T_{i}$ is the ion temperature, $v_{e i}$ is the frequency of ion-electron collisions given by Alexandrov, Bogdankevich, and Rukhadze (1984), Chen (1984) and $\varepsilon_{\mathrm{R}}$ is the reduced amplitude of external weak large-scale electric field in the local Deicer field units. Assuming that all perturbations oscillate with the same frequency, $\omega$, the linearised governing equations can be combined into the modified dispersion relation (MDR) of the form

$$
\sum_{i=0}^{4} P_{i} \Omega^{i}=0
$$

where the coefficients $P_{i}$ are given by Kryshtal, Voitsekhovska, and Gerasimenko (2019), $\Omega \equiv \omega / k_{z} V_{A}$ is the dimensionless frequency, $V_{A}$ is the Alfvén speed, and $k_{z}$ is the longitudinal wave vector component (along the fields $\mathbf{E}_{0} \| \mathbf{B}_{0}$ ). The MDR takes into account spatial inhomogeneity of plasma density and temperature, paired Coulomb collisions and the existence of sub-Dreicer electric field.

We are going to disregard all aperiodic roots of Equation 2. Finding physically acceptable solutions requires a very strict set of constraints on possible values of the plasma parameters. We are interested in weak amplification of waves, therefore the roots to the polynomial in Equation 2 will have to satisfy the condition (see Kryshtal, Voitsekhovska, and Gerasimenko, 2019, for details)

$$
\left.\Gamma_{k} \equiv \frac{\gamma}{\omega}\right|_{\omega=\omega_{k}} \ll 1(k=1, \ldots, 4)
$$

where the index $k$ labels the possible roots of Equation 2, $\omega$ and $\gamma$ are the real and imaginary parts of the roots, respectively. This equation provides a measure of correctness for the use of linear approximation for the determination of growth rate of instability. Previous studies 
by Kryshtal and Gerasimenko (2004), Kryshtal (2005) determined and discussed the growth rate corresponding to the cases $\nabla n \neq 0 ; \nabla T=0$ and $\nabla n=0 ; \nabla T \neq 0$. Clearly, the case we are studying here $(\nabla n \neq 0 ; \nabla T \neq 0)$ is the most general case, and it should not be considered as a linear combination of the above two simpler limits.

The growth rate for the model can be written as (see, e.g., Kryshtal, Voitsekhovska, and Gerasimenko, 2019, where the expressions of the functions $F_{1}$ and $F_{2}$ are also provided)

$$
\left.\Gamma_{k} \equiv \frac{\gamma}{\omega}\right|_{\omega=\omega_{k}}=\left.\sqrt{\frac{\pi}{2}} \frac{\left(\Omega-\beta_{A} \varepsilon_{R}\right)}{\beta \Omega^{2}} \frac{F_{1}}{F_{2}}\right|_{\omega=\omega_{k}} \ll 1,(k=1, \ldots, 4),
$$

where

$$
\beta_{A} \equiv \frac{V_{T e}}{V_{A}}
$$

and $\beta$ is the is plasma-beta parameter.

When determining the realistic solutions of our dispersion relation we took into account a few aspects that are driven by the physical background of the problem. First, in order to determine where the growth rate is positive (i.e. solutions are unstable), we excluded the solutions of the MDR that did not satisfy the criterion given by Equation 3. This restriction is equivalent with not considering fast aperiodic processes of amplification or damping of the waves. Next, from all possible solutions we considered only those which exhibit "signchanged" growth rates. We considered the existence of the dividing curve $\Gamma_{k}=0$ on the surface $\Gamma_{k}=\Gamma\left(z_{i}, k_{*}\right),(k=1, \ldots, 4)$ as possible evidence for the appearance of undamped waves with small amplitudes in pre-flare plasma. Importantly, from an observational point of view, these amplitude values can exceed the level of thermal noise. Such an appearance may be important for the existence of different three-wave interactions in pre-flare plasma and thus forecasting flares in ARs.

\section{Investigation of Kinetic and EM Wave Generation and Stability}

In an earlier paper Kryshtal et al. (2017) studied the modified dispersion relation (MDR) for low-frequency kinetic waves and the wave solutions were identified and classified for magnetic fields in the range $\mathbf{B}_{0} \in(\sqrt{1000}, 1000) \mathrm{G}$, taking into account the influence of the sub-Dreicer electric field, drift plasma motions, paired Coulomb collisions and smallscale Bernstein turbulence when no complete inhomogeneity (density and temperature) was considered. In the same study we identified the combination of plasma parameters that correspond to the loop's footpoint in the chromosphere using the VAL (F) model (Vernazza, Avrett, and Loeser, 1981) at the height of $h=2.429 \mathrm{Mm}$ above the solar surface. These plasma parameters are shown in Table 1, and they will be used in our calculations.

In this paper we use only one value of magnetic field strength $\left|\mathbf{B}_{0}\right|$ for each model of the solar atmosphere. Throughout our calculations we are going to use the value of $\sigma=5$ for the free parameter of the model (see, e.g., Kryshtal, Gerasimenko, and Voitsekhovska, 2012), which allows us to take into account, on the phenomenological level, the contributions of all kinds of mutual collisions of charged particles within the Bhatnagar-Gross-Krook (BGK) integral. Furthermore, we assume that paired Coulomb collisions are the dominant effect in the pre-flare plasma prior to the turbulence appearance. When Bernstein turbulence begins to dominate (the turbulent phase), the collision rate is described by the effective collision 
Table 1 Main physical characteristics of a pre-flare plasma at the chromospheric part of a loop in a solar AR. Here $\omega_{*}$ is the ratio of plasma frequency, $\omega_{p}$, to the ion-cyclotron frequency, $\Omega_{i} ; v_{c} \equiv \sigma v_{e i} / \Omega_{i}$ is the Coulomb collision frequency; $r_{i}$ is the ion-cyclotron gyroradius, $L$ is the density characteristic scale of plasma spatial inhomogeneity. All these values were extracted from the VAL (F) solar atmospheric model. Here we assume that the temperature $(T)$ and number density $(n)$ of ions and electrons are equal. The quantity $\sigma$ in the expression for $\nu_{c}$ plays the role of the free parameter and physically bounded as $1 \leq \sigma \leq \sigma_{\max }$. $\sigma=1$ corresponds to hypothetical situation when the condition for instability development is maximal, i.e. when ion-electron collisions completely dominate. The value $\sigma=\sigma_{\max }$ corresponds to the case when the instability is completely suppressed by the collisions. It has been estimated by, e.g., Kryshtal (2002) and Kryshtal, Gerasimenko, and Voitsekhovska (2012) that in this limiting case $\sigma_{\max }<10$.

\begin{tabular}{|c|c|c|c|c|c|c|c|c|}
\hline $\begin{array}{l}n, \\
10^{9} \mathrm{~cm}^{-3}\end{array}$ & $\begin{array}{l}T, \\
10^{5} \mathrm{~K}\end{array}$ & $\begin{array}{l}\mathrm{h}, \\
\mathrm{km}\end{array}$ & $\begin{array}{l}\left|\mathbf{B}_{0}\right| \\
\mathrm{mT}\end{array}$ & $\omega_{*}$ & $\begin{array}{l}v_{c}, \\
10^{-4}\end{array}$ & $\begin{array}{l}\beta, \\
10^{-4}\end{array}$ & $\begin{array}{l}r_{i}, \\
\mathrm{~cm}\end{array}$ & $\begin{array}{l}L, \\
10^{5} \mathrm{~cm}\end{array}$ \\
\hline 2.57 & 4.47 & 2429 & 3.162 & 5.15 & 4.61 & 3.64 & 4.66 & 1 \\
\hline
\end{tabular}

frequency, $v_{e f f}$, (Galeev et al., 1972), valid if $v_{\text {eff }} \gg \mu \Omega_{i}$ (here, $\mu \equiv \sqrt{m_{e} / m_{i}}$ is electronto-ion mass ratio) and its value is given by

$$
v_{e f f}=\frac{\Gamma}{z_{e}} \Omega_{e},
$$

where $\Gamma$ is the linear reduced growth rate of second quasi-Bernstein harmonics (see, e.g., Kryshtal, Gerasimenko, and Voitsekhovska, 2012), $\Omega_{e}$ is the electron cyclotron frequency and $z_{e}$ is the electron kinetic parameter

$$
z_{e} \equiv \frac{k_{\perp}^{2} V_{T e}^{2}}{\Omega_{e}^{2}}
$$

The problem of the influence of small-scale Bernstein turbulence on low-frequency waves is a very important aspect of our problem and it has been previously investigated by Kryshtal et al. (2017). Their analysis showed that second harmonics of oblique quasiBernstein modes are possible in the range of magnetic field that is characteristic in pre-flare AR chromospheres (from few $\mathrm{mT}$ up to few tens of $\mathrm{mT}$ ). The dispersion relation they derived admitted of analytical solutions that describe the KAW and KIAW,

$$
\begin{gathered}
\Omega_{K A W}= \pm \sqrt{1+z_{i}\left(t_{*}+\frac{3}{4}\right)}, \\
\Omega_{K I A W}= \pm \mu \beta_{A} \sqrt{\frac{1-z_{i}}{1+z_{i} t_{*}}},
\end{gathered}
$$

where the \pm sign stands for forward and backward propagating waves, $z_{i}$ is the ion kinetic parameter defined by

$$
z_{i} \equiv \frac{k_{\perp}^{2} V_{T i}^{2}}{\Omega_{i}^{2}},
$$

with $V_{T i}$ being the ion thermal velocity, $k_{\perp}^{2} \equiv k^{2}-k_{z}^{2}$ the perpendicular component of wave vector, $k_{z}$ the longitudinal component (along the fields $\mathbf{E}_{0} \| \mathbf{B}_{0}$ ), and $t_{*}=T_{e} / T_{i}$ is the ratio of electron and ion temperatures.

We should mention that the nature of the solutions should not be different from the case studied by Kryshtal et al. (2017), however, due to the fact that the collisions are described 
by $v_{\text {eff }} \neq 0$, we expect that the solutions of the MDR (i.e. the roots of the fourth order polynomial given by Equation 2) will be modified in magnitude due to the presence of extra physical effects. That is why the solutions studied here will be modifications of results obtained earlier by Kryshtal et al. (2017).

At the chromospheric height shown in Table 1, using the semi-empirical solar atmospheric VAL (F) model (Vernazza, Avrett, and Loeser, 1981), we have investigated the stability of the four roots of the MDR (Equation 2). Our analysis show that the four roots have different behaviour and they represent the forward and backward propagating modified KAW, and modified KIAW, respectively. Our analysis has been repeated for six different values of magnetic field strength, i.e. $\left|\mathbf{B}_{0}\right|=\sqrt{1000} \approx 31.62 \mathrm{mT} ; 100 \mathrm{mT} ; 300 \mathrm{mT} ; 500 \mathrm{mT}$; $700 \mathrm{mT}$ and $1000 \mathrm{mT}$. Although not all values are strictly adequate for chromospheric conditions, we used this extended magnetic field strength interval to ensure robustness of our results. In addition, solutions to the dispersion relation have been sought so that the parameters of the problem were varied in the intervals

$$
\begin{aligned}
& 5 \cdot 10^{-7} \leq \varepsilon_{R} \leq 10^{-2} \\
& -5 \leq \delta \leq 5 ; \quad\left(\delta= \pm \frac{L}{L_{T}}\right) \\
& 1 \leq \sigma \leq 6 \\
& 0.001 \leq k_{*} \leq 0.25 \\
& 0.001 \leq z_{i} \leq 0.25
\end{aligned}
$$

Here $L_{T}$ is the characteristic scale of inhomogeneity of plasma temperature, $k_{*}=k_{z} / k$. The boundary values of the above parameters are the values at which an instability appears and begins to develop, i.e. when the imaginary part of the frequency changes sign from negative to positive.

Numerical analysis reveals that, for the model we are using, the boundary values that satisfy the imposed physical restrictions occur when $\left(t_{*}\right)_{\text {bound }}=4$ and $\sigma_{\max }=6$, i.e. instabilities with a growth rate that is changing sign satisfying the condition 3 , appear when $t_{*}=4$ and disappear when $\sigma=6$. In our calculations the value of $\delta$ was chosen such that it leads to the most favorable instability development and closest (in absolute value) to the classical value of $\delta=2$ corresponding to the drift-resistive instability (see, e.g., Somov, 1994).

The boundary values for $\varepsilon_{R}, k_{*}$ and $z_{i}$ correspond to the value $\Gamma_{1+}-$ the first positive value of the reduced growth rate in the half-space $\Gamma>0$ after the growth rate has changed its sign from negative to positive. It is easy to show that all terms in the expression of the reduced growth rate given by Equations 4, describing the effect of the drift motions contain the factor

$$
\rho \equiv \frac{\rho_{i}}{L}
$$

In order to estimate the influence of the spatial inhomogeneity in plasma temperature and density on the reduced growth rate, we define the parameter

$$
R_{0 \rho} \equiv \frac{\Gamma_{0}-\Gamma_{\rho}}{\Gamma_{0}},
$$

where $\Gamma_{0} \equiv \Gamma_{1+}$ when $\rho=0$ and $\Gamma_{\rho} \equiv \Gamma_{1+}$ when $\rho \neq 0$.

All the most significant boundary values for the parameters of low-frequency instabilities, which we have investigated for the VAL (F) atmospheric model are presented in Table 2. 
Table 2 The calculated values of the main characteristics of instability of the modified KIAW (the $\Omega_{3}$ and $\Omega_{4}$ roots of the modified dispersion equation) and KAW (the $\Omega_{2}$ root) for the plasma parameters given in Table 1 for the VAL (F) solar atmospheric model. Here $v_{t u r b}$ describes the saturated Bernstein turbulence.

\begin{tabular}{lllr}
\hline Wave type & $(k)_{\text {bound }}$ & $(z)_{\text {bound }}$ & $R_{0 \rho}$ \\
\hline direct KIAW $\left(v=v_{\text {turb }}\right)$ & 0.029 & 0.011 & -1.57 \\
inverse KIAW $\left(v=v_{\text {turb }}\right)$ & -0.0172 & 0.0604 & -1.83 \\
inverse KAW $\left(v=v_{\text {turb }}\right)$ & -0.0132 & 0.011 & -1.48 \\
inverse KIAW $\left(v=v_{c}\right)$ & -0.0035 & 0.125 & -2.55 \\
\hline
\end{tabular}

For the particular case we are investigating we assumed that $\left(t_{*}\right)_{\text {bound }}=4, \sigma=5, \delta=-2$ and $10^{-4} \leq\left(\varepsilon_{R}\right)_{\text {bound }} \leq 1.2 \times 10^{-4}$. In this case, three out of four possible wave types can be generated (Kryshtal et al., 2017). It should be noted that Table 2 contains data for the instabilities which show a sign-change, i.e. when the reduced growth rates given by Equation 4 pass through their zero.

In ARs, at the footpoints of magnetic loops (at heights which correspond to the lowmiddle chromosphere), the generation of second harmonics of Bernstein modes are possible as a result of corresponding instability with has anomalously low threshold of excitation and, therefore, can be transformed into the state of saturated turbulence (see, e.g., Kryshtal, Voitsekhovska, and Gerasimenko, 2019). Hence, the appearance and development of kinetic waves, which have much higher threshold of excitation, may be possible on the background of small-scale saturated Bernstein turbulence. At this stage, Bernstein modes can generate undamped waves (this has been proven in previous studies by Kryshtal, Gerasimenko, and Voitsekhovska, 2012; Kryshtal et al., 2015). In such a situation the appearance of an electromagnetic (EM) wave, - a spike of microwave emission, - the forerunner of a flare, is possible through a nonlinear three-wave interaction (Willes and Robinson, 1996):

$$
B_{1}+B_{2} \rightarrow E M W
$$

where $B_{1}$ and $B_{2}$ represent the Bernstein modes and EMW stands for the transverse electromagnetic wave. In their study Willes and Robinson (1996) have shown that in the case of the above wave interaction the probability of the process is maximal if the $B_{1}$ and $B_{2}$ modes belong to the same morphological class. However, the process of coalescence has an extremely small probability. Therefore, here we investigate the more complicated, yet more realistic outcome for the Bernsteinian phase of the instability, when it transforms into a small-scale turbulence, then reaches a state of saturation, in which kinetic waves will be generated. These kinetic waves (KAW and KIAW) are the necessary participants in a number of three-wave interactions (see, e.g., Yukhimuk et al., 1998, 2000) that, in principle, can produce the EM forerunner of a flare or can results in the depolarisation of solar radio emission (see, e.g., Sirenko et al., 2000). Usually the probability of decay in three-wave interaction is supposed to be much more probable than coalescence. Obviously, in the case when one of the waves participating in this process is propagating with no damping (or both are), this probability can only increase.

In a previous study Kryshtal et al. (2015) investigated the generation and development of the instability of the first harmonic of quasi-Bernstein modes, which actually coincides with upper-hybrid wave (UHW) in the context of our current model. Yukhimuk et al. (1998) have proven that electromagnetic emission in the microwave range can be produced through the process of three-wave interaction of the type

$$
U H W \rightarrow K A W+E M W .
$$


Kryshtal et al. (2015) have shown that the first harmonic of quasi-Bernstein modes can produce small amplitude undamped waves. It is clear that in the case when the same waves are generated through the process of KAW's instability development, three-wave processes governed by Equation 15, become more probable EM waves. The same authors estimated the frequency of the EM emission and have compared it with emission from observations. Other comparisons with observations by, e.g., Bogod and Yasnov (2009), Zharkova et al. (2011) and Kashapova, Meshalkina, and Kisil (2012) have shown good, if not exact agreement with theory. We have to emphasise that kinetic waves are necessary participants in all known plasma processes of nonlinear three-wave interactions, not only because they are possibly responsible for generating the spikes of microwave emission, but also in the mutual transformation of one wave to another (Hasegawa and Chen, 1976a,b; Kryshtal et al., 2014; Lyubchyk and Voitenko, 2014), e.g.,

$$
K A W \rightarrow K A W^{\prime}+K I A W .
$$

The above three-wave interaction scheme means that generation of one kinetic Alfvén wave may lead to the generation of the second one as a result of the cascade process,

$$
\begin{gathered}
K I A W \rightarrow K I A W^{\prime}+K A W, \\
K A W \rightarrow K A W^{\prime}+K A W^{\prime \prime} .
\end{gathered}
$$

Given their properties, kinetic waves can be an excellent candidate for plasma heating, they can depolarize solar EM emission (Sirenko et al., 2000), and can accelerate particles in preflare ARs (Aschwanden, 2005). Thus, the possibility of generation of kinetic waves (KAW and KIAW) on the background of small-scale Bernstein turbulence, which we have shown, seems to be very important, because it gives evidence that small-scale turbulence cannot destroy the process of instability development and the time of generation of kinetic waves may be long enough for a number of three-wave processes to be realised.

\section{Results and Discussion}

Our study was devoted to the analysis of the excitation and development of low-frequency instabilities in fully ionised pre-flaring chromospheric plasma. The plasma parameters (density and temperature) were given by the VAL (F) solar atmospheric model.

In Figure 1 (top left panel) we plot the reduced growth rate of instability, $\Gamma$, as a function of $k_{*}$ for the considered VAL (F) solar atmospheric model. In this case the reduced values of effective turbulence and Coulomb collision frequencies satisfy the inequality

$$
v_{t u r b} \gg v_{c} \text {. }
$$

The value of $R_{0 \rho}$ demonstrates that corrections to the induced growth rate $\Gamma$ that take into account the spatial inhomogeneities of plasma temperature and density may be negligibly small in absolute values, but the relative change of $\Gamma$ may be considerable enough.

As can be seen from Figure 1, among the four roots of the modified dispersion relation, only three (direct/inverse KIAW and inverse KAW for the plasma with Coulomb conductivity and small-scale Bernstein turbulence) are unstable and have alternating signs. The root which corresponds to the direct KAW is stable. Therefore, there is a fundamental difference between the present study and Kryshtal, Voitsekhovska, and Gerasimenko (2019), where the 

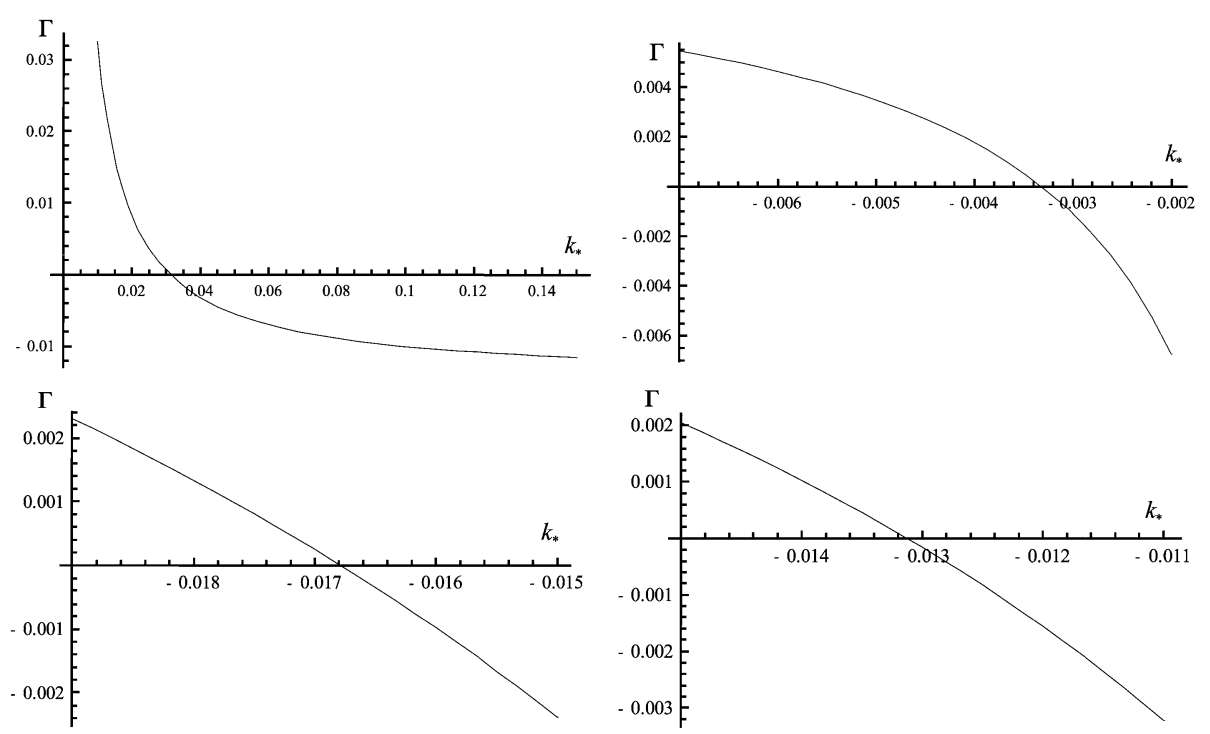

Figure 1 The reduced growth rates of instability, $\Gamma$, for a VAL (F) solar atmospheric model as a function of $k_{*}=k_{z} / k$. The four different cases shown here are: direct KIAW (top left panel); inverse KIAW (top right) for the plasma with Coulomb conductivity; inverse KIAW (bottom left) and inverse KAW (bottom right), both for the plasma with small-scale Bernstein turbulence.

VAL (F) model does not include the unstable roots of the modified dispersion relation. This result was obtained with complete coincidence of the entire "calculated part" (i.e., by taking into account similar approximations and equations presented in Kryshtal, Voitsekhovska, and Gerasimenko (2019), but by applying different values of the parameters of the wave disturbance. The physical parameters we worked with can be considered as typical for the pre-flare stage and instabilities which may appear in plasma during the pre-flare phase are non-beam instabilities.

Our results show that in the current circuit of the loop near its footpoint in the active region, the generation of kinetic waves is possible as a result of the development of an instability during the flare process, when the electron thermal velocity is greater than the current velocity, that is, before the appearance of Buneman instability and beginning of the preliminary heating phase. For semi-empirical solar atmosphere models (including the VAL (F) model) there is an area at the magnetic loop footpoint where instability threshold for the second quasi-Bernstein harmonic is very low (Kryshtal, Gerasimenko, and Voitsekhovska, 2012; Kryshtal et al., 2017).

We have shown that the development of instability of kinetic waves is possible not only in a plasma with Coulomb conductivity, but also in the presence of Bernstein turbulence, i.e. this turbulence will not destroy the generation of kinetic waves. Therefore, waves may have enough time for participation in the three-wave interactions, which may generate electromagnetic wave (Yukhimuk et al., 1998). Observation of these waves can be considered as necessary condition for short-term flare forecast in ARs.

Using standard methods outlined in the paper we derived a dispersion relation in the form of a quartic polynomial whose roots represent two KAWs and two KIAWs propagating in opposite direction. Low-frequency instability can spontaneously develop when paired Coulomb collisions dominate but also when the small-scale Bernstein turbulence is dominant. Among these roots (for the chosen plasma parameters) only the direct KAW shows 
stable behaviour as can be seen from Table 2. For the considered case we have found a slow instability with sufficient low threshold (but much higher than the threshold of excitation of the second quasi-Bernstein harmonic). The instability recovered here is important as it appears in the loop footpoint when traditional drivers of instabilities, i.e. beams of energetic particles are not present (see, e.g., Battaglia, Fletcher, and Simões, 2014).

Our calculations have shown that the spatial inhomogeneity of plasma temperature and density can influence the growth rate of the instability through the values of the drift frequency. Although the absolute value of changes in the reduced growth rate remain very small, the relative changes of the growth rate may be considerable enough to notably influence the solutions of the modified dispersion relation.

The kinetic wave generated due to the instability was suitable enough to determine the influence of the drift plasma motion on the value of the growth rate, because the root corresponding to this wave has a growth rate which changes sign, and its first positive value in the half-space $\Gamma\left(z_{i}, k_{*}\right)>0$ is the best candidate for comparing the cases that correspond to the growth rate.

The problem of generation of the kinetic waves is important in studying the physical processes in a pre-flare plasma because these waves have their own electric field, which allows them, in principle, to play an important role in the pre-flare acceleration of the particles and plasma heating through the generation of turbulence and abnormal resistance. In contrast to MHD instabilities, which have been frequently observed in AR loops, kinetic instabilities and kinetic plasma waves involve spatial and temporal scales (see, e.g., Hasegawa and Chen, 1976a; Galeev and Sudan, 1983, 1985) that are impossible to observe with the current instruments. However, it has been shown that the generation of kinetic waves increases the probability of three-wave processes which will, in turn, produce EM waves which can be detected by observers and thus may become an early warning signal of high energy processes in a pre-flare AR (Fárník and Savy, 1998; Schmahl et al., 1986; Willes and Robinson, 1996).

Núñez et al. (2005) pointed out that the flare forecasting precision can be increased by combining synoptical and causal forecast, where the synoptical forecast is based on the morphology of ARs before a flare. The causal forecast is based on knowledge of the physical mechanisms of the flaring process. The results presented in this paper may be considered as only necessary part of the causal forecast.

Acknowledgements OC would like to thank the Ukrainian Scientific and Technical Center, PN 6060; Integrated Scientific Programme of the National Academy of Science of Ukraine on Space Research. VF, GV and IB thank The Royal Society, International Exchanges Scheme, collaboration with Chile (IE170301) and Brazil (IES $\backslash \mathrm{R} 1 \backslash 191114$ ). VF and GV are grateful to the Science and Technology Facilities Council (STFC) grant ST/M000826/1 for support provided. This research is also partially funded by the European Union's Horizon 2020 research and innovation program under grant agreement No. 824135 (SOLARNET). This work also greatly benefited from the discussions at the ISSI workshop "Towards Dynamic Solar Atmospheric Magneto-Seismology with New Generation Instrumentation".

Disclosure of Potential Conflicts of Interest The authors declare that they have no conflicts of interest.

Publisher's Note Springer Nature remains neutral with regard to jurisdictional claims in published maps and institutional affiliations.

Open Access This article is licensed under a Creative Commons Attribution 4.0 International License, which permits use, sharing, adaptation, distribution and reproduction in any medium or format, as long as you give appropriate credit to the original author(s) and the source, provide a link to the Creative Commons licence, and indicate if changes were made. The images or other third party material in this article are included in the article's Creative Commons licence, unless indicated otherwise in a credit line to the material. If material is not included in the article's Creative Commons licence and your intended use is not permitted by statutory regulation or exceeds the permitted use, you will need to obtain permission directly from the copyright holder. To view a copy of this licence, visit http://creativecommons.org/licenses/by/4.0/. 


\section{References}

Alexandrov, A., Bogdankevich, L., Rukhadze, A.: 1984, Principles of Plasma Electrodynamics, Springer, Berlin.

Artemyev, A.V., Zimovets, I., Rankin, R.: 2016, Astron. Astrophys. 589, A101. DOI.

Aschwanden, M.J.: 2001, Astrophys. J. 560, 1035. DOI.

Aschwanden, M.J.: 2005, Physics of the Solar Corona. An Introduction with Problems and Solutions, 2nd edn.

Aurass, H.: 1994, In: Rusin, V., Heinzel, P., Vial, J.-C. (eds.) Solar Coronal Structures, IAU Colloq. 144, 251.

Battaglia, M., Fletcher, L., Simões, P.J.A.: 2014, Astrophys. J. 789, 47. DOI.

Benka, S.G.: 1994 In: Proceedings of Kofu Symposium, 225.

Bogod, V.M., Yasnov, L.V.: 2009, Solar Phys. 255, 253. DOI.

Brodin, G., Stenflo, L., Shukla, P.K.: 2006, Solar Phys. 236, 285. DOI.

Charikov, Y.E., Shabalin, A.N.: 2015, Geomagn. Aeron. 55, 1104. DOI.

Chen, F.F.: 1984, Introduction to Plasma Physics and Controlled Fusion, Volume 1: Plasma Physics, Springer, Berlin.

Dreicer, H.: 1959, Phys. Rev. 115, 238. DOI.

Dreicer, H.: 1960, Phys. Rev. 117, 329. DOI.

Druett, M., Scullion, E., Zharkova, V., et al.: 2017, Nat. Commun. 8, 15905. DOI.

Fárník, F., Savy, S.K.: 1998, Solar Phys. 183, 339. DOI.

Fontenla, J.M., Avrett, E.H., Loeser, R.: 1993, Astrophys. J. 406, 319. DOI.

Foukal, P., Hinata, S.: 1991, Solar Phys. 132, 307. DOI.

Galeev, A.A., Sudan, R.N.: 1983, Handbook of Plasma Physics. Vol. 1: Basic Plasma Physics I.

Galeev, A.A., Sudan, R.N.: 1985, Handbook of Plasma Physics. Vol. 2: Basic Plasma Physics II.

Galeev, A.A., Lominadze, D.G., Pataraya, A.D., Segdeev, R.Z., Stepanov, K.N.: 1972, ZhETF Pisma Redaktsiiu 15, 417.

Griem, H.R.: 1974, Spectral Line Broadening by Plasmas.

Guo, J., Emslie, A.G., Piana, M.: 2013, Astrophys. J. 766, 28. DOI.

Harra, L.K., Matthews, S.A., Culhane, J.L.: 2001, Astrophys. J. Lett. 549, L245. DOI.

Hasegawa, A., Chen, L.: 1976a, Phys. Rev. Lett. 36, 1362. DOI.

Hasegawa, A., Chen, L.: 1976b, Phys. Fluids 19, 1924. DOI.

Heinzel, P., Dorotovič, I., Rutten, R.J.: 2007, The Physics of Chromospheric Plasmas 368.

Heyvaerts, J., Priest, E.R., Rust, D.M.: 1977, Astrophys. J. 216, 123. DOI.

Holman, G.: 1985, Astrophys. J. 293, 584. DOI.

Kashapova, L.K., Meshalkina, N.S., Kisil, M.S.: 2012, Solar Phys. 280, 525. DOI.

Kryshtal, A.N.: 2002, J. Plasma Phys. 68, 137. DOI.

Kryshtal, A.N.: 2005, J. Plasma Phys. 71, 729. DOI.

Kryshtal, A.N., Gerasimenko, S.V.: 2004, Astron. Astrophys. 420, 1107. DOI.

Kryshtal, A.N., Gerasimenko, S.V., Voitsekhovska, A.D.: 2012, Adv. Space Res. 49, 791. DOI.

Kryshtal, A.N., Gerasimenko, S.V., Voitsekhovska, A.D.: 2014, Astrophys. Space Sci. 349, 637. DOI.

Kryshtal, A.N., Voitsekhovska, A.D., Gerasimenko, S.V.: 2019, Kinemat. Phys. Celest. Bodies 35, 105. DOI.

Kryshtal, A.N., Gerasimenko, S.V., Voitsekhovska, A.D., Cheremnykh, O.K.: 2014, Kinemat. Phys. Celest. Bodies 30, 147. DOI.

Kryshtal, A., Fedun, V., Gerasimenko, S., Voitsekhovska, A.: 2015, Solar Phys. 290, 3331. DOI.

Kryshtal, A.N., Voitsekhovska, A.D., Gerasimenko, S.V., Cheremnykh, O.K.: 2017, Kinemat. Phys. Celest. Bodies 33, 149. DOI.

Kuijpers, J., van der Post, P., Slottje, C.: 1981, Astron. Astrophys. 103, 331.

Kumar, P., Nakariakov, V.M., Cho, K.-S.: 2016, Astrophys. J. 822, 7. DOI.

Lyubchyk, O., Voitenko, Y.: 2014, Ann. Geophys. 32, 1407. DOI.

Machado, M.E., Avrett, E.H., Vernazza, J.E., Noyes, R.W.: 1980, Astrophys. J. 242, 336. DOI.

Miller, J.A., Cargill, P.J., Emslie, A.G., Holman, G.D., Dennis, B.R., LaRosa, T.N., et al.: 1997, J. Geophys. Res. 102, 14631. DOI.

Moghaddam-Taaheri, E., Goertz, C.K.: 1990, Astrophys. J. 352, 361. DOI.

Núñez, M., Fidalgo, R., Baena, M., Morales, R.: 2005, Ann. Geophys. 23, 3129. DOI.

Reale, F.: 2010, Living Rev. Solar Phys. 7, 5. DOI.

Reznikova, V.E., Melnikov, V.F., Shibasaki, K., Gorbikov, S.P., Pyatakov, N.P., Myagkova, I.N., Ji, H.: 2009, Astrophys. J. 697, 735. DOI.

Schmahl, E.J., Webb, D.F., Woodgate, B., Waggett, P., Bentley, R., Hurford, G., et al.: 1986, In: Kundu, M., Woodgate, B. (eds.) Energetic Phenomena on the Sun.

Shchukina, N.G., Trujillo Bueno, J.: 2013, In: Kosovichev, A.G., de Gouveia Dal Pino, E., Yan, Y. (eds.) Solar and Astrophysical Dynamos and Magnetic Activity, IAU Symposium 294, 107. DOI. 
Sirenko, O., Voitenko, Y., Goossens, M., Yukhimuk, A.: 2000, In: Verheest, F., Goossens, M., Hellberg, M.A., Bharuthram, R. (eds.) Waves in Dusty, Solar, and Space Plasmas, American Institute of Physics Conference Series 537, 287. DOI.

Somov, B.V.: 1994, Fundamentals of Cosmic Electrodynamics 191. DOI.

Tsap, Y.T., Kopylova, Y.G.: 2017, Geomagn. Aeron. 57, 996.

Tsiklauri, D.: 2011, Phys. Plasmas 18, 092903. DOI.

Vernazza, J.E., Avrett, E.H., Loeser, R.: 1981, Astrophys. J. Suppl. 45, 635. DOI.

Voitenko, Y., Goossens, M.: 2002, Solar Phys. 206, 285. DOI.

Willes, A.J., Robinson, P.A.: 1996, Astrophys. J. 467, 465. DOI.

Wu, D.J., Fang, C.: 2007, Astrophys. J. 659, L181. DOI.

Xu, Y., Emslie, A.G.: 2007, Bull. Am. Astron. Soc. 39, 213.

Yukhimuk, V., Voitenko, Y., Fedun, V., Yukhimuk, A.: 1998, J. Plasma Phys. 60, 485. DOI.

Yukhimuk, A., Fedun, V., Sirenko, O., Voitenko, Y.: 2000, In: Verheest, F., Goossens, M., Hellberg, M.A., Bharuthram, R. (eds.) Waves in Dusty, Solar, and Space Plasmas, American Institute of Physics Conference Series 537, 311. DOI.

Zaitsev, V.V., Stepanov, A.V.: 2018, Geomagn. Aeron. 58, 831. DOI.

Zaitsev, V.V., Stepanov, A.V., Tsap, Y.T.: 1994, Kinemat. Fizika Nebesnykh Tel. 10, 3.

Zharkova, V.V., Kashapova, L.K., Chornogor, S.N., Andrienko, O.V.: 2011, Mon. Not. Roy. Astron. Soc. 411, 1562. DOI. 